\title{
Estimating the Eddy Viscosity Profile from Velocity Spirals in the Ekman Boundary Layer
}

\author{
$\operatorname{AUTHOR}(\mathrm{S}):$
}

Yoshikawa, Yutaka; Endoh, Takahiro

\section{CITATION:}

Yoshikawa, Yutaka ... [et al]. Estimating the Eddy Viscosity Profile from Velocity Spirals in the Ekman Boundary Layer. Journal of Atmospheric and Oceanic Technology 2015, 32(4): 793-804

ISSUE DATE:

2015-04

URL:

http://hdl.handle.net/2433/203023

\section{RIGHT:}

(c) Copyright 2015 American Meteorological Society (AMS).; The full-text file will be made open to the public on 18 June 2015 in accordance with publisher's 'Terms and Conditions for Self-Archiving'. 


\title{
Estimating the Eddy Viscosity Profile from Velocity Spirals in the Ekman Boundary Layer
}

\author{
YUTAKA YOSHIKAWA \\ Graduate School of Science, Kyoto University, Kyoto, Japan \\ TAKAHIRO ENDOH \\ University of Tokyo Ocean Alliance, University of Tokyo, Tokyo, Japan
}

(Manuscript received 29 April 2014, in final form 18 December 2014)

\begin{abstract}
Turbulent mixing induced by tidal currents near the sea bottom plays a key role in coastal and shallow sea environments. Many attempts have been made to quantify turbulent mixing near the seabed, such as velocity microstructure measurements with microstructure profilers and turbulent Reynolds stress measurements using acoustic Doppler current profilers (ADCPs). This study proposes an alternative method in which the Ekman balance equations are solved with measured velocity spirals to estimate the eddy viscosity profile. Three schemes (schemes 1,2, and 3) are described in this paper; schemes 1 and 2 were used in previous studies, while scheme 3 is newly proposed in the present study. The performance of the three schemes was tested using velocity spirals simulated with an idealized eddy viscosity profile, showing that scheme 2 is useful if the random measurement errors are small, while scheme 3 is useful when the errors in the Ekman balance are small. The performance was also evaluated using measured velocity spirals. This method utilizes velocity measured with standard ADCPs operated in normal modes, allowing for easier and more frequent quantifications of the mixing averaged over longer periods.
\end{abstract}

\section{Introduction}

Strong tidal currents in shallow seas are accompanied by intense vertical shear near the sea bottom. This shear induces turbulent mixing and vertical turbulent fluxes of the momentum, heat, and water properties (e.g., Thorpe 2007). Hydrographic and biogeochemical structures near the sea bottom therefore depend on the boundary layer mixing. Quantification of the mixing structure near the seafloor is thus required for better understanding of coastal and shallow sea environments.

Many attempts have been made to quantify the mixing structure near the sea bottom. Microstructure profilers (MSPs), which estimate kinetic energy dissipation rates (Osborn 1974; Lueck et al. 2002), are frequently used to estimate eddy diffusivity (e.g., Osborn 1980). In general, the intermittency of the turbulence is large, and

Corresponding author address: Yutaka Yoshikawa, Graduate School of Science, Kyoto University, Kitashirakawa-Oiwake, Sakyo, Kyoto 606-8502, Japan.

E-mail: yosikawa@kugi.kyoto-u.ac.jp determining the mean dissipation rates and mean eddy diffusivity requires a large number of statistically independent measurements (Burchard et al. 2008). This makes MSP measurement laborious when estimating long-term averages of the mixing. Acoustic Doppler current profilers (ADCPs) can be used to measure the turbulent Reynolds stress from (high frequency) velocity variances in two different directions (Lohrmann et al. 1990; Lu and Lueck 1999; Stacey et al. 1999; Rippeth et al. 2003). Because this method (the variance method) relies on differences in the velocity variances, measurement errors (noise) included in the variance can easily contaminate the stress estimations, unless the errors are exactly equal in the two variances (Burchard et al. 2008). Measurement noise can be reduced by increasing the number of acoustic pings (e.g., Nidzieko et al. 2006), though this will reduce the measurement period because of the limited capacity of the battery. Thus, estimation of the Reynolds stress is often limited to short periods (e.g., a few days).

In this study, an alternative method for estimating longer-term turbulent mixing structure is discussed. The 
method utilizes the fact that the mean velocity profiles are determined by the mean turbulent mixing (the eddy viscosity) in the bottom boundary layer, where the turbulent mixing term is dominant in the momentum equations. This method (referred to as the profile method) was first used by Soulsby (1990). He used the harmonic coefficients of the dominant tidal $\left(M_{2}\right)$ current and its momentum equations and estimated eddy viscosity at several levels. However, the estimated eddy viscosity showed a large degree of scatter, and the eddy viscosity profile could not be discussed in a quantitative manner. Recently, Yoshikawa et al. (2010) analyzed the harmonic coefficients of semidiurnal and diurnal tidal currents as well as a steady current in the bottom Ekman boundary layer, and estimated the eddy viscosity profiles by solving the Ekman balance equations using the least squares method. The estimated eddy viscosity was largest about $5 \mathrm{~m}$ from the sea bottom, and decreased almost exponentially above that level. The magnitude of the eddy viscosity was larger in spring tide than in neap tide. These features are consistent with numerical simulations of tide-induced turbulence near the sea bottom (e.g., Sakamoto and Akitomo (2008)).

One reason for successful estimations of the eddy viscosity profile in Yoshikawa et al. (2010) seems to be the use of the least squares method. However, as described in section 2, one could say that Soulsby (1990) also used the least squares method, though the quantity to be minimized was different from that in Yoshikawa et al. (2010). This demonstrates that the performance of the least squares method depends greatly on the selected quantity to be minimized. It is therefore worth investigating whether there are other least squares methods that may provide a more accurate estimation of the mixing structure near the seabed.

In this study, we compare three schemes for estimating eddy viscosity. The first scheme corresponds to that of Soulsby (1990), the second scheme follows that of Yoshikawa et al. (2010), and the third scheme is newly proposed in this study. These schemes are described in section 2. Using velocity spirals simulated with an idealized eddy viscosity profile, the performance of the three schemes is investigated in section 3 . The schemes are also evaluated using measured velocity spirals in section 4 , followed by concluding remarks in section 5 .

\section{Estimation schemes}

The estimation schemes are outlined as follows. We assume that the harmonic coefficients of the tidal currents are known as a function of height $z$. Using the complex notation of the horizontal velocity vector $(w=u+\iota v)$, a tidal current of frequency $\omega$ is represented as

$$
w(z, t)=W(z) \exp \iota \omega t,
$$

where $t$ is time. In this notation, the tidal current is decomposed into counterclockwise-rotating $(\omega>0)$ and clockwise-rotating $(\omega<0)$ current components. The conventional tidal harmonic coefficients represented by amplitudes $(U$ and $V)$ and phases $\left(\lambda_{U}\right.$ and $\left.\lambda_{V}\right)$ of the zonal and meridional velocities, respectively, are converted to the real and imaginary parts of the complex amplitude $W$ of the counterclockwise $(\omega>0)$ and clockwise $(\omega<0)$ components as

$$
\begin{aligned}
W(z)= & \frac{U(z) \cos \lambda_{U}(z) \pm V(z) \sin \lambda_{V}(z)}{2} \\
& +\iota \frac{V(z) \cos \lambda_{V}(z) \mp U(z) \sin \lambda_{U}(z)}{2} \text { for } \omega \gtrless 0 .
\end{aligned}
$$

For a steady constituent $(\omega=0)$, there are only two parameters (the real and imaginary parts of $W$ ) that correspond to the zonal and meridional velocities of the current.

The three schemes are based on the Ekman boundary layer equations,

$$
\frac{\partial w}{\partial t}+\iota f w=\frac{\partial}{\partial z}\left[\mu(z) \frac{\partial w}{\partial z}\right],
$$

where $f$ is the Coriolis parameter and $\mu(z)$ is the eddy viscosity to be estimated. The boundary layer components that should obey the above-mentioned boundary layer equations need to be calculated a priori from the measured velocity. If the currents are barotropic in an interior layer (above the boundary layer), then the components can be easily estimated by subtracting the velocity at the top of the boundary layer as

$$
W(z)=\tilde{W}(z)-\tilde{W}(H+\delta),
$$

where $W$ is the boundary layer component, $\tilde{W}$ is the total component (boundary plus interior components), $H$ is the boundary layer height, and $\delta$ is an adjustment parameter. Here, $\delta$ (set as a few meters) is required to avoid errors in the eddy viscosity estimation near $z=H$.

Inserting Eq. (1) into Eq. (2) yields

$$
\iota(f+\omega) W(z)=\frac{\partial}{\partial z}\left[\mu(z) \frac{\partial}{\partial z} W(z)\right] .
$$

Note that Eq. (3) contains a total of four equations (equations for both positive and negative $\omega$, each having both real and imaginary parts) for one tidal component (e.g., $M_{2}$ ). For the steady constituent, there are two 
equations. Thus, the number of equations $N$ is $4 \times M+2$, where $M$ is the number of tidal constituents to be analyzed. If the diurnal and semidiurnal tidal constituents as well as the steady constituent are analyzed, then $N$ equals 10 . These equations can be used to estimate $\mu(z)$ at each level $z$. Because $N$ is larger than the number of quantities to be estimated $[\mu(z)]$, the least squares technique can be used. The question is, "which quantity is to be minimized." In this study, three schemes are investigated in which different quantities are minimized.

Before discussing each scheme in detail, Eq. (3) is discretized in the vertical as

$$
\underbrace{\iota\left(f+\omega_{n}\right) W_{k+1 / 2, n} \Delta z}_{a_{k, n}}=\mu_{k+1} \underbrace{\frac{W_{k+3 / 2, n}-W_{k+1 / 2, n}}{\Delta z}}_{s_{k+1, n}}-\mu_{k} \underbrace{\frac{W_{k+1 / 2, n}-W_{k-1 / 2, n}}{\Delta z}}_{s_{k, n}},
$$

where $n$ represents the tidal constituent (e.g., $n=1$ is the clockwise semidiurnal constituent; $n=2$ is the counterclockwise semidiurnal constituent, ...), $k$ represents the vertical level, $W_{k+1 / 2, n}$ is the complex tidal harmonic coefficient at $z_{k+1 / 2}$, and $\mu_{k}$ is the eddy viscosity at $z_{k}$. The velocity and eddy viscosity are defined on a staggered grid. For simplicity, the acceleration and velocity shear are denoted by $a_{k, n}$ and $s_{k, n}$, respectively.

\section{a. Scheme 1}

Vertical integration of Eq. (4) from $z_{k}$ to $z_{K}=H$ yields

$$
\underbrace{\sum_{k^{\prime}=k}^{K-1} a_{k^{\prime}, n}}_{t_{k, n}}=\mu_{K} s_{K, n}-\mu_{k} s_{k, n},
$$

where the vertical integration of $a_{k, n}$ corresponding to the momentum stress difference between $z_{k}$ and $H$ is represented as $t_{k, n}$ for simplicity. If $\mu_{K}$ is known, then dividing the real and the imaginary parts of Eq. (5) by the real and the imaginary parts of $s_{k, n}$, respectively, yields a total of $N \mu_{k} \mathrm{~s}$, and averaging them results in a unique $\mu_{k}$ at each $z_{k}$. This scheme basically corresponds to the method used by Soulsby (1990), though the Ekman boundary layer equations were not used in his study. Note that the above-mentioned ensemble averaging $\left(\mu_{k}=\sum_{n=1}^{N} \mu_{k, n} / N\right)$ corresponds to the least squares solution that minimizes the error $\varepsilon_{k, n}$ included in each ensemble $\mu_{k, n}=\mu_{k}+\varepsilon_{k, n}$. Thus, this scheme can also be regarded as a least squares method.

The value of $\mu_{K}$ remains arbitrary. Usually, the eddy viscosity at the top of the boundary layer is much smaller than that within the layer, so $\mu_{K}=0$ is a good approximation and is assumed in the following for this scheme. This scheme is referred to as scheme 1 .

\section{b. Scheme 2}

This scheme (referred to as scheme 2) was used by Yoshikawa et al. (2010). In this scheme, errors are assumed in the momentum Eq. (4),

$$
a_{k, n}=\mu_{k+1} s_{k+1, n}-\mu_{k} s_{k, n}+\varepsilon_{k, n} .
$$

Note that $\varepsilon_{k, n}$ is a complex number. The sum of the squared $\varepsilon_{k, n}$ is defined as

$$
E=\sum_{k, m}\left(\varepsilon_{k, m} \mid \varepsilon_{k, m}\right),
$$

where $(a \mid b)=\left(a b^{*}+a^{*} b\right) / 2\left(a^{*}\right.$ is the complex conjugate of $a$ ). The minimum $E$ is obtained if

$$
\begin{aligned}
\frac{1}{2} \frac{\partial E}{\partial \mu_{K}}= & \sum_{n}\left(s_{K, n} \mid s_{K, n}\right) \mu_{K}-\sum_{n}\left(s_{K, n} \mid s_{K-1, n}\right) \mu_{K-1} \\
& -\sum_{n}\left(a_{K, n} \mid s_{K, n}\right)=0 \\
\frac{1}{2} \frac{\partial E}{\partial \mu_{k}}= & \sum_{n}\left(s_{k+1, n} \mid s_{k, n}\right) \mu_{k+1}-2 \sum_{n}\left(s_{k, n} \mid s_{k, n}\right) \mu_{k} \\
& +\sum_{n}\left(s_{k-1, n} \mid s_{k, n}\right) \mu_{k-1}=0 \quad(2 \leq k \leq K-1) \\
\frac{1}{2} \frac{\partial E}{\partial \mu_{1}}= & \sum_{n}\left(s_{2, n} \mid s_{1, n}\right) \mu_{2}-\sum_{n}\left(s_{1, n} \mid s_{1, n}\right) \mu_{1} \\
& -\sum_{n}\left(a_{2, n} \mid s_{1, n}\right)=0
\end{aligned}
$$

are satisfied. The above-mentioned equations are solved to obtain $\mu_{k}$ for $k=1, \ldots, K$. Although $\mu_{K}=0$ was assumed in Yoshikawa et al. (2010), the number of equations $(N \times K)$ is always larger than the number of unknown quantities $(K)$ and hence $\mu_{K}$ can be estimated.

In the above-mentioned equations, the acceleration term $\left(a_{k, m}\right)$ appears only at the top $(k=K)$ and bottom $(k=1)$ levels. Thus, this scheme does not rely on the measured profiles of the acceleration or velocity (except at $k=1$ and $k=K$ ) but on the vertical shear profile $s_{k, m}$. The ensemble average is taken such that a larger weight is given to the constituent with the larger shear.

\section{c. Scheme 3}

This scheme (referred to as scheme 3 ) is newly proposed in this study. In this scheme, a vertical integration 
of Eq. (4) is performed as in scheme 1. However, in contrast to scheme 1 , the errors to be minimized are assumed in the integrated equations as

$$
t_{k, n}=\mu_{K} s_{K, n}-\mu_{k} s_{k, n}+\varepsilon_{k, n} .
$$

The least squares technique yields

$$
\begin{aligned}
\frac{1}{2} \frac{\partial}{\partial \mu_{K}} E= & (K-1) \sum_{n}\left(s_{K, n} \mid s_{K, n}\right) \mu_{K}-\sum_{k=1}^{K-1} \sum_{n}\left(s_{K, n} \mid s_{k, n}\right) \mu_{k} \\
& -\sum_{k=1}^{K-1} \sum_{n}\left(t_{k, n} \mid s_{K, n}\right)=0,
\end{aligned}
$$$$
\frac{1}{2} \frac{\partial}{\partial \mu_{k}} E=\sum_{n}\left(s_{k, n} \mid s_{k, n}\right) \mu_{k}-\sum_{n}\left(s_{K, n} \mid s_{k, n}\right) \mu_{K}
$$$$
+\sum_{n}\left(t_{k, n} \mid s_{k, n}\right)=0 . \quad(1 \leq k \leq K-1) .
$$

These equations are solved to obtain $\mu_{k}$.

The eddy viscosity derived from this scheme is easily interpreted if $\mu_{K}=0$. In this case, Eqs. (9) and (10) reduce to

$$
\mu_{k}=-\frac{\sum_{n}\left(t_{k, n} \mid s_{k, n}\right)}{\sum_{n}\left(s_{k, n} \mid s_{k, n}\right)},
$$

showing that $\mu_{k}$ is the ratio between the stress $t_{k, n}$ in the direction of the shear vector $s_{k, n}$ and the magnitude of the shear. The ensemble average is taken such that a larger weight is given to the constituent with the larger shear.

\section{Validation using simulated velocity spirals with an idealized eddy viscosity profile}

In this section, the three schemes explained in the previous section are tested using velocity spirals simulated with an idealized (given) eddy viscosity profile. Taking into account the observations of Yoshikawa et al. (2010) and numerical experiments of Sakamoto and Akitomo (2008), we set the eddy viscosity as

$$
\begin{aligned}
\mu= & \left\{\left[\kappa U_{*}(z+0.1)\right]^{-1}+\left[5 \exp \left(\frac{z}{0.3 \kappa U_{*} / f}\right)\right]^{-1}\right\}^{-1} \\
& +10^{-6} \mathrm{~m}^{2} \mathrm{~s}^{-1}
\end{aligned}
$$

where $U_{*}=2.5 \times 10^{-3} \mathrm{~m} \mathrm{~s}^{-1}$ and $\kappa=0.4$. The boundary layer component $W(z)$ of the tidal current of frequency $\omega_{n}$ is calculated by solving Eq. (3) with the following boundary conditions:

$$
\begin{gathered}
\frac{\partial W}{\partial z}=0 \quad \text { at } \quad z=Z, \\
W=-W_{i} \quad \text { at } \quad z=0,
\end{gathered}
$$

where $W_{i}$ is the tidal harmonic coefficient in the interior layer, and $Z(=50 \mathrm{~m})$ is a certain level in the interior $(Z>H)$. The calculated velocity is sampled at $z_{k+1 / 2}$ and is used as $W_{k+1 / 2, n}$. The sampling interval is set as $1 \mathrm{~m}$ as in standard ADCP measurements. In the following, a steady constituent with $W_{i}=1.0 \mathrm{~m} \mathrm{~s}^{-1}$ is used to examine the performance of the estimation schemes against measurement errors (section 3a) and errors in the Ekman balance equations (section 3b). The effects of the selected boundary layer height $(H)$ are also examined with this steady constituent (section 3c). Finally, the semidiurnal $\left(\omega= \pm 1.41 \times 10^{-4} \mathrm{~s}^{-1}\right)$ and diurnal $(\omega=$ $\left.\pm 6.76 \times 10^{-5} \mathrm{~s}^{-1}\right)$ constituents and the steady constituent are used to examine the effects of the number of tidal constituents on the eddy viscosity estimations (section 3d).

\section{a. Sensitivity to measurement error}

In actual observations, measurements are likely to be contaminated by errors. In this subsection, the performance of each scheme under random measurement errors is investigated with the boundary layer height $(H)$ being set as $25 \mathrm{~m}$.

Random errors are added to the entire profiles of the simulated velocity. The standard deviation of the error $(\Delta W)$ is set as $\Delta W=1.0 \times 10^{-4}, 1.0 \times 10^{-3}$, and $1.0 \times 10^{-2} \mathrm{~m} \mathrm{~s}^{-1}$ in this subsection. A total of 32 different velocity profiles were created with different random errors for each $\Delta W$, and the mean and standard deviation of 32 samples of the estimated eddy viscosity were calculated. A sample of the velocity profile with random measurement errors is shown in Fig. 1. The error becomes apparent for $\Delta W=1.0 \times 10^{-2} \mathrm{~m} \mathrm{~s}^{-1}$.

The eddy viscosity estimated with scheme 1 (Fig. 2a) differs from the true one at around $z=19 \mathrm{~m}$ even for $\Delta W=1.0 \times 10^{-4} \mathrm{~m} \mathrm{~s}^{-1}$. Around this level, the vertical gradient of the zonal velocity component $\left[\operatorname{Re}\left(s_{k, n}\right)\right]$ is close to zero (black solid line in Fig. 1). As a result, eddy viscosity estimated from this component $\left\{\mu_{k, n} \simeq\right.$ $\left.\operatorname{Re}\left(t_{k, n}\right) /\left[\operatorname{Re}\left(s_{k, n}\right)+\epsilon\right]\right\}$ is more likely to be contaminated by small measurement error $(\epsilon)$. In fact, the error at this level does not become zero even for $\Delta W=0$ because of finite difference errors (the estimated eddy viscosity becomes negative due to the finite difference errors, and the negative eddy viscosity is replaced by $0.1 \mathrm{~m}^{2} \mathrm{~s}^{-1}$ in Fig. 2). As $\Delta W$ increases, the difference between the estimated and true eddy viscosity profiles becomes large at levels of low signal-to-noise ( $\mathrm{SN}$ ) ratios. The standard deviation of the estimated eddy viscosity also increases as $\Delta W$ increases, indicating that the estimation becomes more uncertain.

The eddy viscosity estimated with scheme 2 (Fig. 2b) almost coincides with the true one for 


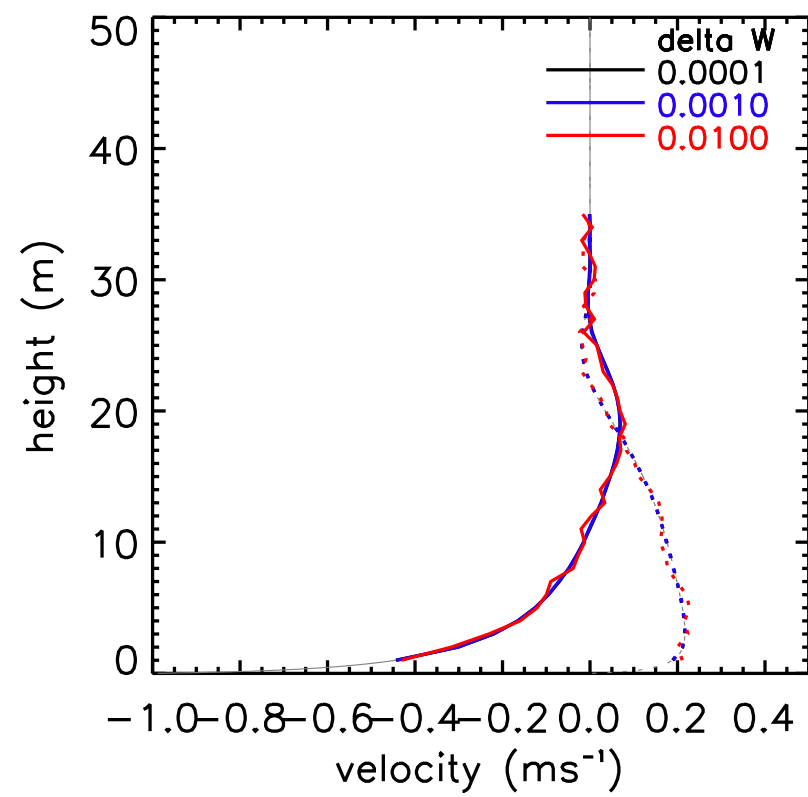

FIG. 1. Velocity profiles (steady current). Zonal (real) and meridional (imaginary) components are represented by solid and dotted lines, respectively. Color (black, blue, and red) represents velocity profiles sampled at 1-m-depth interval, whose magnitude of the measurement noise $\Delta W$ is denoted in top-right corner of the figure. (The black and blue lines almost coincide.) Gray lines show true velocity profiles calculated with higher vertical resolution.

$\Delta W=1.0 \times 10^{-4} \mathrm{~m} \mathrm{~s}^{-1}$. However, for $\Delta W \geq 1.0 \times 10^{-3} \mathrm{~m} \mathrm{~s}^{-1}$, a systematic underestimation in the eddy viscosity appears. This is because $\sum_{n}\left(s_{k, n}, s_{k, n}\right)$ in Eq. (7) increases more rapidly than $\sum_{n}\left(s_{k \pm 1, n}, s_{k, n}\right)$ as the measurement error increases (because the measurement errors in $s_{k \pm 1, n}$ and $s_{k, n}$ are canceled out when ensemble averaging). Thus, scheme 2 is weak against the random measurement errors. It should be noted, however, that smoothing of the velocity profile before calculating $a_{k, n}$ and $s_{k, n}$ is quite effective in reducing the systematic underestimation (not shown).

The eddy viscosity with scheme 3 (Fig. 2c) is very close to the true one even for $\Delta W=1.0 \times 10^{-3} \mathrm{~m} \mathrm{~s}^{-1}$. The eddy viscosity is slightly underestimated for $\Delta W=1.0 \times 10^{-2} \mathrm{~m} \mathrm{~s}^{-1}$ for reasons similar to those mentioned for scheme 2 , but the degree of the underestimation is smaller. This is because scheme 3 relies on the vertically integrated velocity (in which random measurement errors tend to be canceled out), while scheme 2 relies on velocity shear (where measurement errors are exaggerated). All these results show that scheme 3 is the most robust scheme against the random measurement errors.

\section{b. Sensitivity to error in the Ekman balance}

The Ekman balance is assumed in the present methods; hence, a deviation from the balance can be a source of
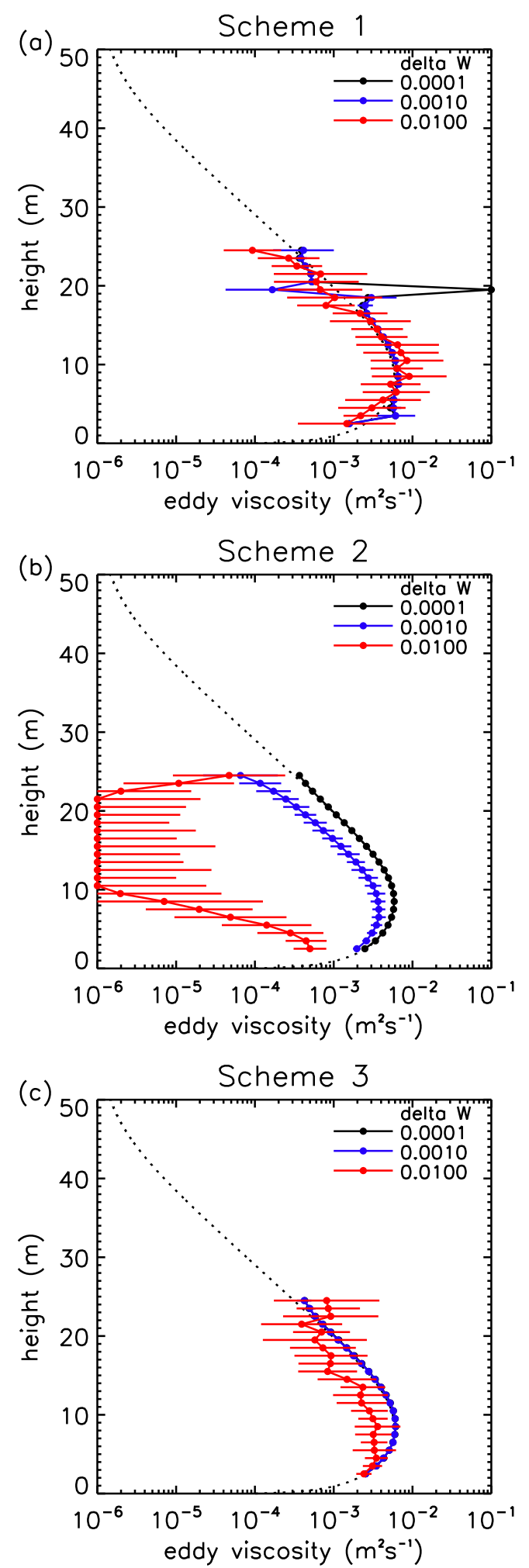

FIG. 2. Eddy viscosity profiles estimated with (a) scheme 1, (b) scheme 2, and (c) scheme 3. Also shown are $\Delta W=1.0 \times 10^{-4} \mathrm{~m} \mathrm{~s}^{-1}$ (black line), $1.0 \times 10^{-3} \mathrm{~m} \mathrm{~s}^{-1}$ (blue line), and $1.0 \times 10^{-2} \mathrm{~m} \mathrm{~s}^{-1}$ (red line). Black dotted line shows true (given) eddy viscosity. Eddy viscosity values smaller than $1.0 \times 10^{-6}$ or larger than $0.1 \mathrm{~m}^{2} \mathrm{~s}^{-1}$ are replaced with these values. 
errors. An advection term that is partly balanced with the acceleration term in the left-hand side of Eq. (3) can be such a source. In this subsection, the effects of this error (referred to as a balance error) are examined. Here, the measurement error $(\Delta W)$ is absent and the boundary layer height $(H)$ is set to $25 \mathrm{~m}$ as in the previous subsection.

The balance error is expected to exist around a certain level rather than be distributed randomly over the whole boundary layer. Here, we assume that the balance error occurs at $z=10 \mathrm{~m}$ or $z=20 \mathrm{~m}$. A random balance error is added on the left-hand side of Eq. (3). A total of 32 sets of equations with random balance errors at $z=10 \mathrm{~m}$ or $z=20 \mathrm{~m}$ are solved to obtain 32 sets of eddy viscosity profiles. The standard deviation of the errors is given by $f \Delta B$ with $\Delta B=1.0 \times 10^{-2}, 1.0 \times 10^{-1}$, and $1.0 \mathrm{~m} \mathrm{~s}^{-1}$.

Figure 3 shows the mean and standard deviation of the eddy viscosity with $\Delta B$ given at $z=10 \mathrm{~m}$. The eddy viscosity estimated with scheme 1 (Fig. 3a) deviates from the true one below $10 \mathrm{~m}$ (note that the deviation around $z=19 \mathrm{~m}$ is due to the finite difference errors mentioned in the previous subsection). Because of the vertical integration of Eq. (4), the balance error contaminates $a_{k, n}$ below $10 \mathrm{~m}$ and induces an error at those levels. The estimation errors in schemes 2 and 3 (Figs. 3b,c) are much smaller than those in scheme 1 . This shows the general robustness of these schemes relative to scheme 1 .

If the balance error exists in the upper part of the boundary layer, then the performance of schemes 2 and 3 slightly changes. Figure 4 shows the mean and standard deviation of the eddy viscosity with $\Delta B$ given at $z=20 \mathrm{~m}$. The eddy viscosities estimated with scheme 1 (Fig. 4a) deviate from the true values below $20 \mathrm{~m}$ (as expected). In scheme 3 , the deviations are much larger at $z=20 \mathrm{~m}$ than at $z=10 \mathrm{~m}$, because the velocity magnitude (and hence the SN ratio) is smaller at $z=20 \mathrm{~m}$ than at $z=10 \mathrm{~m}$ (Fig. 1). Vertical integration of Eq. (4) helps in spreading the balance error effects downward as in scheme 1. On the other hand, the errors in scheme 2 are small below $z=20 \mathrm{~m}$ (Fig. 4b). Thus, scheme 2 can perform better than schemes 3 and 1 if the balance error exists at the upper levels of the boundary layer.

\section{c. Sensitivity to selected boundary layer height}

To calculate the Ekman boundary layer components, the boundary layer height $H$ needs to be given a priori. To evaluate the dependence on the given $H$, the eddy viscosity curves estimated with $H=15$ and $35 \mathrm{~m}$ as well as $H=25 \mathrm{~m}$ are shown in Fig. 5. Here, we assume $\Delta W=1.0 \times 10^{-4} \mathrm{~m} \mathrm{~s}^{-1}$ and $\Delta B=1.0 \times 10^{-2} \mathrm{~m} \mathrm{~s}^{-1}$ at $z=10 \mathrm{~m}$.

For $H=15 \mathrm{~m}$, both schemes 2 and 3 show a slight overestimation at $10 \mathrm{~m} \leq z_{k} \leq 15 \mathrm{~m}$, while scheme 1
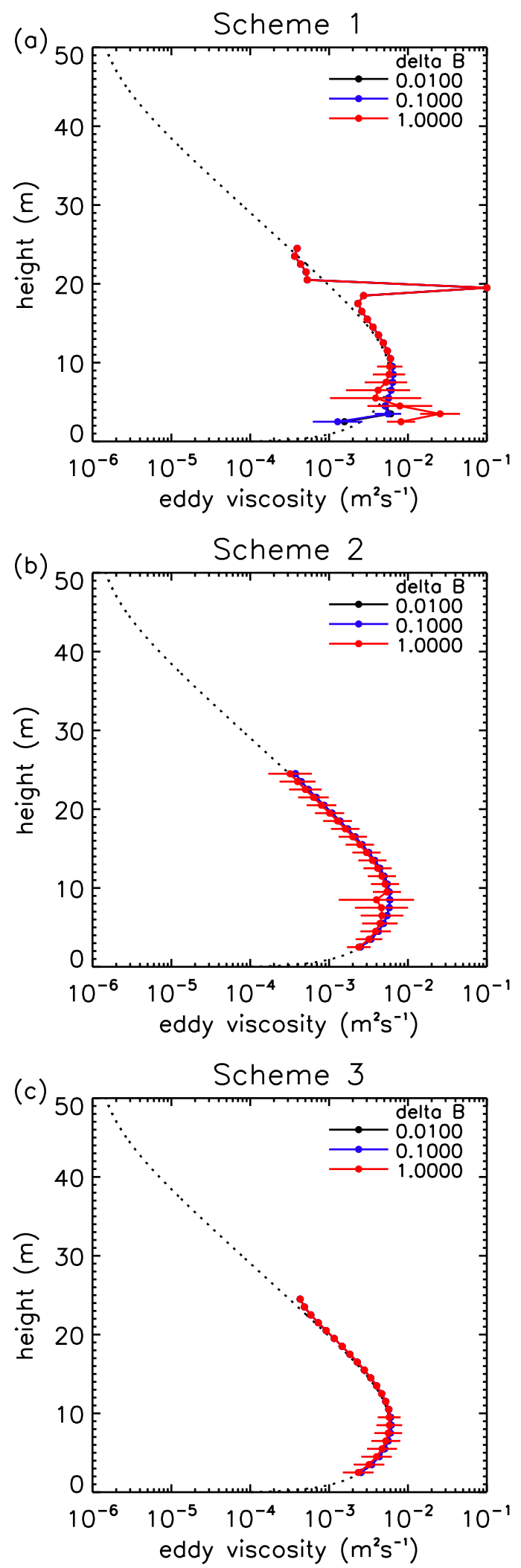

FIG. 3. As in Fig. 2, but $\Delta B=1.0 \times 10^{-2} \mathrm{~m} \mathrm{~s}^{-1}$ (black line), $1.0 \times 10^{-1} \mathrm{~m} \mathrm{~s}^{-1}$ (blue line), and $1.0 \mathrm{~m} \mathrm{~s}^{-1}$ (red line) given at $z=10 \mathrm{~m}$. 

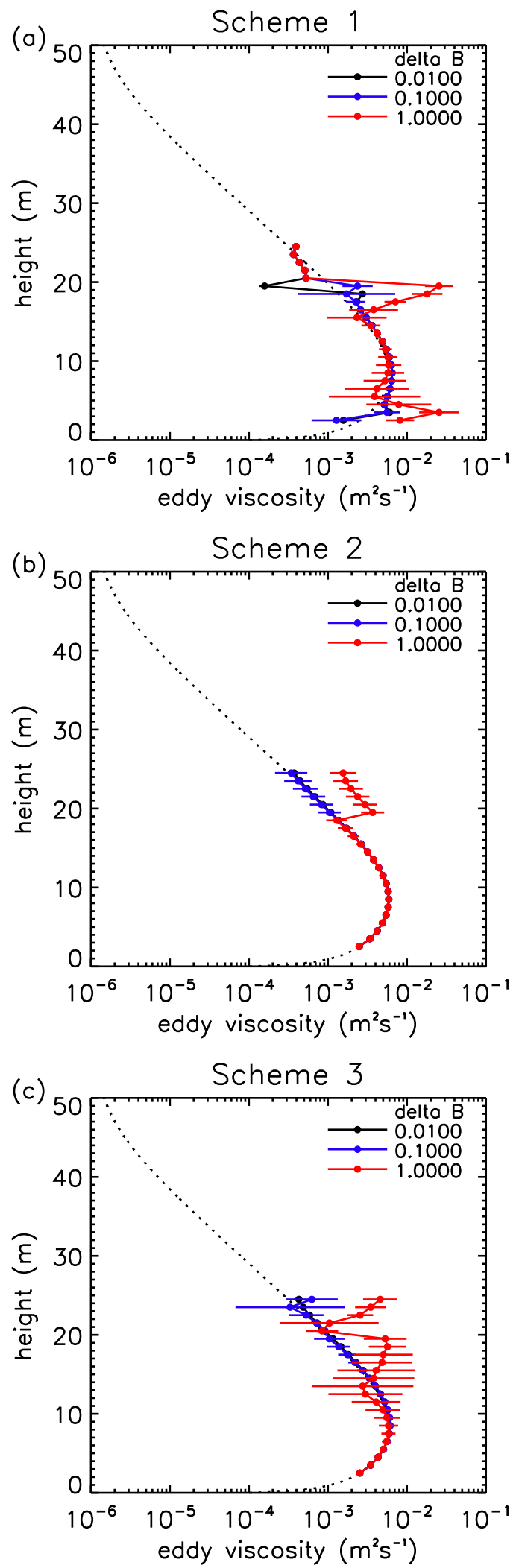

FIG. 4. As in Fig. 3, except that $\Delta B$ is given at $z=20 \mathrm{~m}$. apparently fails to reproduce the true eddy viscosity. For $H=35 \mathrm{~m}$, estimations of all schemes are uncertain above about $30 \mathrm{~m}$, where the velocity is quite small and the SN ratio is low. Note that regardless of the choice of $H$, the estimated eddy viscosity with schemes 2 and 3 converges to the true value at depths where the velocity amplitude is significant. This means that $H$ does not greatly affect the eddy viscosity estimated at those levels. In practice, an appropriate value of $H$ can be found by setting it as slightly larger than the highest level of the significant velocity magnitude and decreasing it until the estimated eddy viscosity profile becomes stable.

\section{d. Sensitivity to the number of tidal constituents}

Finally, the number of tidal constituents to be used is changed. In the previous subsections, a steady constituent with $W_{i}=1.0 \mathrm{~m} \mathrm{~s}^{-1}$ is used. Here, the semidiurnal $\left(\omega= \pm 1.41 \times 10^{-4} \mathrm{~s}^{-1}\right)$ and diurnal $\left(\omega= \pm 6.76 \times 10^{-5} \mathrm{~s}^{-1}\right)$ tidal constituents as well as the steady constituent are used for the eddy viscosity estimation. The amplitudes of each constituent are listed in Table 1 . The diurnal constituent is artificially set weak in order to evaluate the performance of the estimation schemes with minor tidal constituents added in the analysis.

Figure 6 shows the eddy viscosity estimated from the steady constituent only $(N=2)$; the steady and semidiurnal constituents $(N=6)$; and the steady, semidiurnal, and diurnal constituents $(N=10)$. Here we set $H=25 \mathrm{~m}, \Delta W=1.0 \times 10^{-3} \mathrm{~m} \mathrm{~s}^{-1}$, and $\Delta B=0.1 \mathrm{~m} \mathrm{~s}^{-1}$ at $z=20 \mathrm{~m}$. Values of $\Delta W$ and $\Delta B$ smaller than these values show less dependence on $N$. It is clear that increasing the number of tidal constituents does not necessarily result in a more accurate estimation. The estimation error in scheme 1 spreads in the vertical as $N$ increases. This is because the number of levels at which the velocity gradient becomes small increases as $N$ increases. [Note that the gradient $s_{k, n}$ (rather than the sum of the gradient $\left.\sum_{n} s_{k, n}\right)$ is used in the denominator in scheme 1.] In scheme 2, the systematic underestimation of the eddy viscosity becomes larger as $N$ increases, because the coefficient $\sum_{n}\left(s_{k \pm 1, n}, s_{k, n}\right) / \sum_{n}\left(s_{k, n}, s_{k, n}\right)$ becomes smaller as $N$ increases. Note that the systematic underestimation disappears for all $N$ for $\Delta W=1.0 \times 10^{-3} \mathrm{~m} \mathrm{~s}^{-1}$ (not shown). Thus, if the measurement errors are small, then $N$ does not greatly affect the estimation of scheme 2 . The eddy viscosity estimated with scheme 3 has a smaller standard deviation with a larger number of tidal constituents. The larger $N$, however, induces larger errors in the estimated eddy viscosity for $\Delta W=1.0 \times 10^{-2} \mathrm{~m} \mathrm{~s}^{-1}$ (not shown). Thus, schemes 2 and 3 are less affected by the number of tidal constituents if the measurement error is sufficiently small. This is because these schemes give a larger weight 

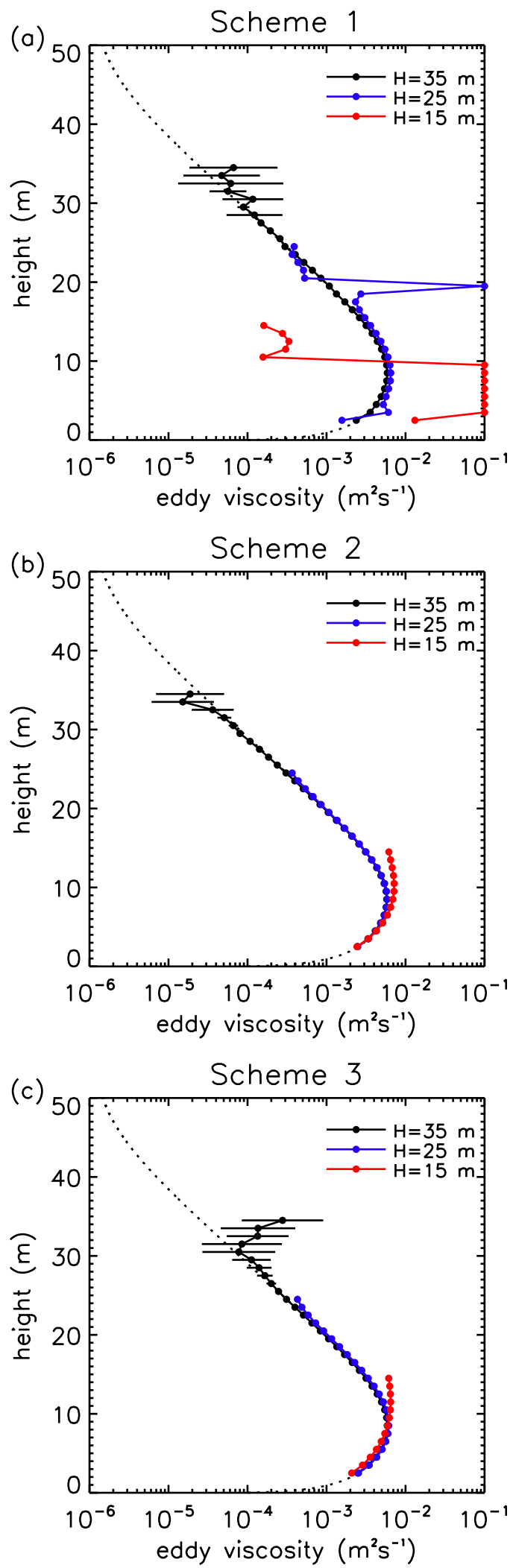

FIG. 5. As in Fig. 2, but $H=35 \mathrm{~m}$ (black line), $25 \mathrm{~m}$ (blue line), and $15 \mathrm{~m}$ (red line); $\Delta W=1.0 \times 10^{-4} \mathrm{~m} \mathrm{~s}^{-1}$ and $\Delta B=1.0 \times 10^{-2} \mathrm{~m} \mathrm{~s}^{-1}$ given at $z=10 \mathrm{~m}$
TABLE 1. Internal velocity $W_{i}$ of steady, semidiurnal, and diurnal constituents.

\begin{tabular}{lccc}
\hline \hline & $\begin{array}{c}\omega \\
\left(10^{-4} \mathrm{~s}^{-1}\right)\end{array}$ & $\begin{array}{c}\text { Real } \\
\left(\mathrm{m} \mathrm{s}^{-1}\right)\end{array}$ & $\begin{array}{c}\text { Imaginary } \\
\left(\mathrm{m} \mathrm{s}^{-1}\right)\end{array}$ \\
\hline Steady & 0.0 & 1.0 & 0.0 \\
Semidiurnal & 1.41 & 0.0 & 0.2 \\
Diurnal & -1.41 & 0.0 & 1.0 \\
& 0.676 & 0.05 & 0.0 \\
& -0.676 & 0.1 & 0.0 \\
\hline
\end{tabular}

to the constituent with the larger shear (sections $2 \mathrm{~b}$ and c). They are, however, affected if the measurement error is large. Thus, the most appropriate value of $N$ depends on the scheme used and the errors included in the data. A practical way to find the best scheme and $N$ is described in the next section.

\section{Evaluation using observed velocity spirals}

In this section, ADCP data of Yoshikawa et al. (2010) are used to examine the performance of the three estimation schemes mentioned above. The ADCPs were deployed at two stations in the East China Sea (ECS) shelf. Station $1\left(31^{\circ} 45^{\prime} \mathrm{N}, 127^{\circ} 25^{\prime} \mathrm{E}\right)$ is located near the shelf break where the mean water depth is $128 \mathrm{~m}$. At this station, an ADCP (RDI, Workhorse $300 \mathrm{kHz}$ ) was deployed during August 19-October 17 (60 days) in 2008. Station $2\left(31^{\circ} 45^{\prime} \mathrm{N}, 125^{\circ} 30^{\prime} \mathrm{E}\right)$ is located near the center of the ECS shelf where the mean water depth is $60 \mathrm{~m}$. At this station, an ADCP (RD Instruments, Workhorse $600 \mathrm{kHz}$ ) was deployed during 18-24 July (6.5 days) in 2009. At both stations, an ADCP was deployed from the training ship Nagasaki Maru of Nagasaki University. At station 1 (2), velocities were measured from a height of $4 \mathrm{~m}(2 \mathrm{~m})$ to $80-110 \mathrm{~m}(30-50 \mathrm{~m})$ above the bottom with a 2-m $(1 \mathrm{~m})$ bin size. Both ADCPs were set in a trawlresistant bottom mount (Floating Technology, AL200) to minimize damage from trawling by fishing boats. The acoustic pings were pulsed continuously with less than a 3-s interval. The resultant nominal measurement error of the hourly velocity was less than $1.0 \times 10^{-3} \mathrm{~m} \mathrm{~s}^{-1}$. Tidal harmonic analysis was performed on these hourly velocity data. For the 2008 data, a total of 29 tidal components were analyzed. For the 2009 data, a total of only six tidal components were analyzed due to the short measurement period. The record lengths of 60 days (1440 hourly velocities) at station 1 and 6 days (144 hourly velocities) at station 2 reduced the measurement errors to $1.0 \times 10^{-3} / \sqrt{1440}=2.6 \times 10^{-5} \mathrm{~m} \mathrm{~s}^{-1}$ at station 1 and $1.0 \times 10^{-3} / \sqrt{144}=8.3 \times 10^{-5} \mathrm{~m} \mathrm{~s}^{-1}$ at station 2 . The magnitude of the measurement errors relative to the largest tidal constituent amplitude at station $1\left(2.6 \times 10^{-5} / 0.15=1.7 \times 10^{-4}\right)$ and station 2 

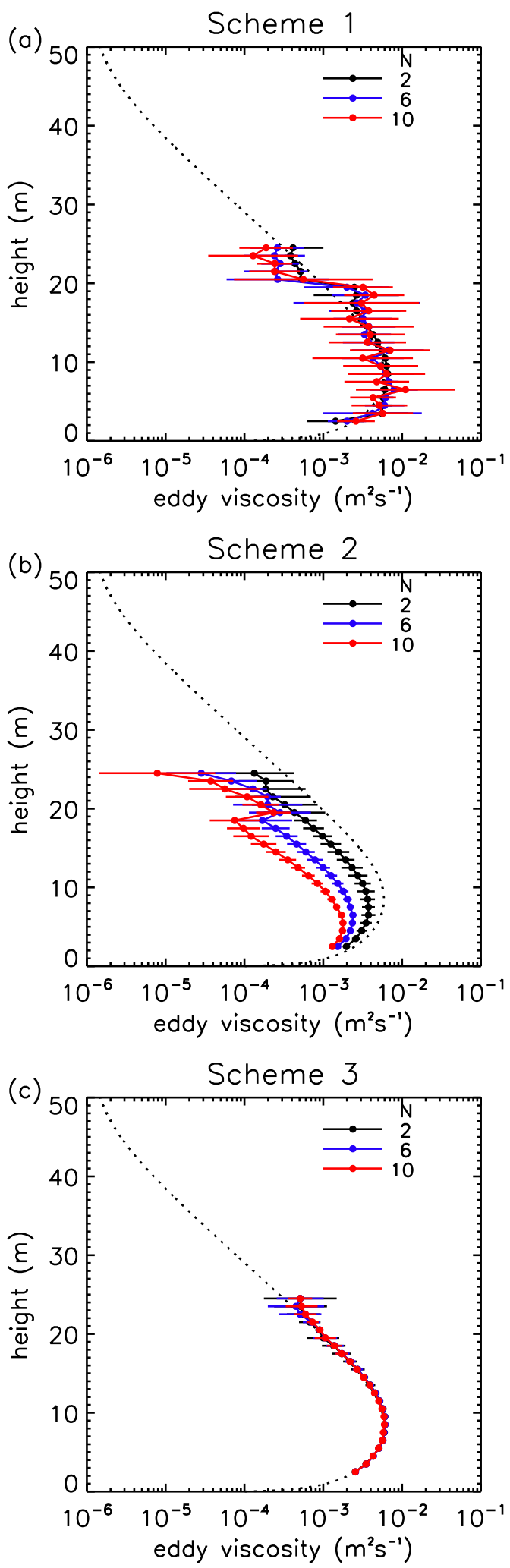

FIG. 6. As in Fig. 2, but estimated with a steady constituent only $(N=2$, black line); steady and semidiurnal constituents $(N=6$, blue line); and steady, semidiurnal, and diurnal constituents $(N=10$ red line). Values $\Delta W=1.0 \times 10^{-3} \mathrm{~m} \mathrm{~s}^{-1}$ and $\Delta B=0.1 \mathrm{~m} \mathrm{~s}^{-1}$ given at $z=20 \mathrm{~m}$. $\left(8.3 \times 10^{-5} / 0.51=1.6 \times 10^{-4}\right)$ is small for both stations, indicating a minor effect of the measurement error. Detailed tidal and mean current features obtained from these ADCPs can be found in Yoshikawa et al. $(2010,2012)$.

Figure 7 shows the eddy viscosity profiles estimated with scheme 1 (black lines), 2 (blue lines), and 3 (red lines) at stations 1 and 2 from the observed velocity profiles (Fig. 8) using two sets of estimation parameters ( $H=30 \mathrm{~m}$ or $25 \mathrm{~m}$ and $N=2$ or 10$)$. Note that the eddy viscosity near the bottom cannot be estimated because no velocity measurements were obtained there.

At both stations, the eddy viscosity estimated with scheme 1 shows an unsmooth profile with unrealistically large $\left(\geq 0.1 \mathrm{~m}^{2} \mathrm{~s}^{-1}\right)$ values at many heights, indicating that scheme 1 is not useful. On the other hand, the eddy viscosity estimated with scheme 2 shows a smooth profile with reasonable values. Some eddy viscosity profiles estimated with scheme 3 are irregular, though others are close to the profiles estimated with scheme 2 . The stable estimation of scheme 2 is reasonable because of the small measurement errors.

The eddy viscosity profiles estimated with scheme 2 with $H=25 \mathrm{~m}$ (crosses) and $H=35 \mathrm{~m}$ (circles) are almost the same at both stations, indicating that $H$ in this range does not affect the estimation. The eddy viscosity, estimated with the steady, semidiurnal, and diurnal constituents $(N=10$, solid line $)$, is slightly smaller than that estimated with the clockwise-rotating semidiurnal constituent (the largest constituent) only $(N=2$, dotted line). This may indicate a small effect of the measurement errors (section 3d).

On the other hand, the eddy viscosity estimated with scheme 3 depends on the estimation parameters. At station 1 , the eddy viscosity profiles estimated with $N=10$ (solid lines) are irregular between 15- and 20-m height, while the profiles estimated with $N=2$ (dotted lines) are smooth with reasonable values below $25 \mathrm{~m}$. At station 2, on the other hand, eddy viscosity profiles estimated with $N=10$ (solid lines) are smooth, while those with $N=2$ (dotted lines) have an unrealistic local minimum at around 15-m height.

Because the true eddy viscosity profile is unknown, other quantities need to be compared for validation of the eddy viscosity estimations. Here, velocity profiles were reproduced from the Ekman equation (3) with the estimated eddy viscosity profile and compared with the observed profiles for this validation. Figure 8 shows the reproduced velocity profiles of the clockwise semidiurnal constituent (the largest constituent). Note that the velocity is not reproduce if there is an unreasonably large $\left(\geq 0.1 \mathrm{~m}^{2} \mathrm{~s}^{-1}\right)$ value of eddy viscosity in its profile. Thus, scheme 1 and scheme 3 with some $N$ and $H$ parameters were excluded from this validation. 

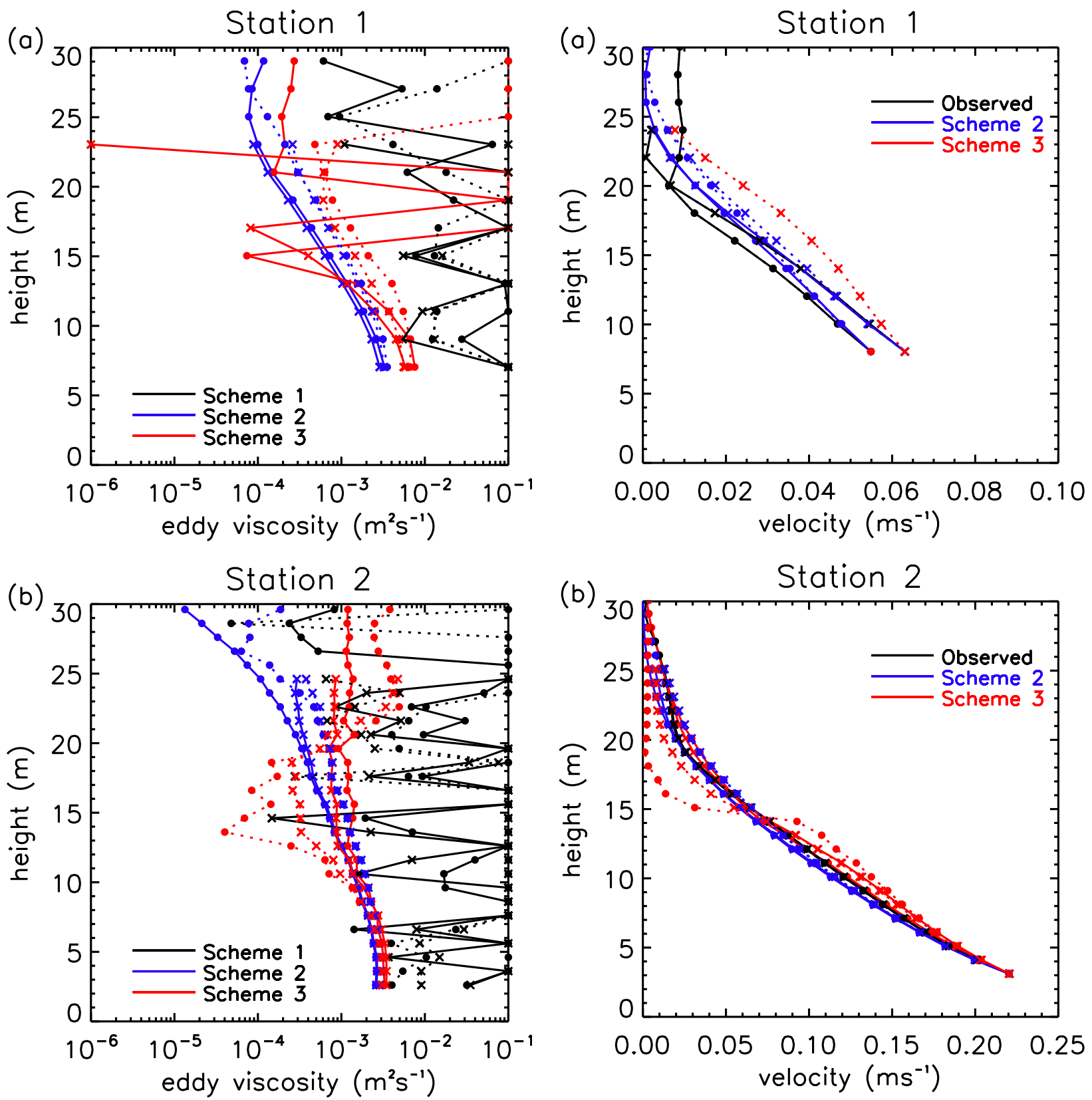

FIG. 7. Eddy viscosity profiles estimated from ADCP data obtained at (a) station 1 and (b) station 2 in the ECS. Black, blue, and red lines denote schemes 1,2 , and 3 , respectively. Solid (dotted) lines show eddy viscosity estimated with $N=10(N=2)$. Lines with circles (crosses) denote eddy viscosity estimated with $H=30 \mathrm{~m}(H=25 \mathrm{~m})$.

At both stations, the velocity reproduced with the eddy viscosity of scheme 2 is closer to the observed one than that of scheme 3 . This demonstrates that scheme 2 provides the best estimates in the present case. The difference in the reproduced and observed velocities means that the acceleration term calculated with the observed velocity [left-hand side of Eq. (3)] does not

FIG. 8. Profiles of the velocity magnitude of the clockwiserotating semidiurnal tidal constituent. Black lines denote the observed profile, and blue and red lines represent velocity reproduced with eddy viscosity estimated with schemes 2 and 3, respectively. Type of line (solid or dotted) and symbols (circles or crosses) are the same as those in Fig. 7.

match the estimated Reynolds stress divergence [righthand side of Eq. (3)]. This suggests that other forcing term(s) should be included in Eq. (3). Thus, this difference can be considered as a measure of the balance error. The balance error seems relatively large at around 20-m height at both stations. This level corresponds to the boundary layer top where horizontal advection effects 
are expected to be large. In fact, Endoh et al. (2014), manuscript submitted to Geophys. Res. Lett.) found large temporal variations in stratification and turbulence due to horizontal advection of different stratification at around this level at station 2. Such temporal variations in mixing can distort the momentum balance from the Ekman one (e.g., Price et al. 1986) and can be a source of the balance error. This could be the main reason why the estimations of scheme 3 are worse than those of scheme 2 (section $3 b$ ).

Judging from the root-mean-square (RMS) difference between the reproduced and observed velocities, the eddy viscosity estimated with scheme 2 using only the clockwise semidiurnal constituent is found to be the best estimation. Thus, the RMS difference between the observed and reproduced velocity can be used to select the optimal scheme and parameters.

\section{Concluding remarks}

In the present study, estimation methods for the eddy viscosity profile from observed profiles of tidal harmonics were investigated. The Ekman boundary layer components and the Ekman balance equations are used. Three estimation schemes were examined: scheme 1 corresponds to the method used in Soulsby (1990), scheme 2 was used by Yoshikawa et al. (2010), and scheme 3 is newly proposed in this study. Sensitivity analysis using the simulated velocity spirals shows that scheme 2 is useful if the measurement error is small, while scheme 3 is useful if the balance error is small. For ADCP data observed in the ECS shelf, the balance error is large; therefore, the eddy viscosity profile estimated with scheme 2 was the most reliable profile. Note that the relative magnitude of the balance errors and the measurement errors will depend on the ADCP setting, measurement period, and measurement location. Therefore, scheme 3 should be kept as a promising scheme for eddy viscosity estimation. As shown in section 4 , both schemes should be applied with several values of $H$ and $N$, and the validity of the eddy viscosity profiles should be examined using velocity profiles reproduced with the estimated eddy viscosity.

It should be noted that the method provides a mean profile of turbulent mixing rather than an instantaneous one. If the monthly-mean velocity is used, then the monthly-mean turbulent mixing is estimated. For this reason, the present method is suited to quantify the "mean" mixing structure, which is important information when discussing longer-term changes (e.g., seasonal changes) of the boundary mixing. Another important aspect of this method is its easy applicability; velocity data obtained with standard ADCPs operated in normal modes can be used, as long as the boundary layer components (Ekman spirals) are identified in the data. The present method can also be applied to the surface boundary layer where the eddy viscosity profiles were estimated from the observed mean Ekman spirals (e.g., Chereskin 1995; Yoshikawa et al. 2007). We believe that these features make the present method useful in extending our understanding of marine turbulent boundary mixing.

Acknowledgments. The authors express their hearty thanks to Kousuke Aoyama, who helped in the preliminary analysis of this work. This work was partly supported by the Japan Society for the Promotion of Science Grants-in-Aid for Scientific Research (22340140, 25287123) and by the joint research program of the Research Institute for Applied Mechanics, Kyushu University.

\section{REFERENCES}

Burchard, H., and Coauthors, 2008: Observational and numerical modeling methods for quantifying coastal ocean turbulence and mixing. Prog. Oceanogr., 76, 399-442, doi:10.1016/ j.pocean.2007.09.005.

Chereskin, T. K., 1995: Direct evidence for an Ekman balance in the California Current. J. Geophys. Res., 100, 18 261-18269, doi:10.1029/95JC02182.

Lohrmann, A., B. Hackett, and L. P. Loed, 1990: High resolution measurements of turbulence, velocity and stress using a pulseto-pulse coherent sonar. J. Atmos. Oceanic Technol., 7, 19-37, doi:10.1175/1520-0426(1990)007<0019:HRMOTV>2.0.CO;2.

Lu, Y., and R. G. Lueck, 1999: Using a broadband ADCP in a tidal channel. Part II: Turbulence. J. Atmos. Oceanic Technol., 16, 1568-1579, doi:10.1175/1520-0426(1999)016<1568: UABAIA $>2.0 . \mathrm{CO} ; 2$.

Lueck, R. G., F. Wolk, and H. Yamazaki, 2002: Oceanic velocity microstructure measurement in the 20th century. J. Oceanogr., 58, 153-174, doi:10.1023/A:1015837020019.

Nidzieko, N. J., F. A. Fong, and J. L. Hench, 2006: Comparison of Reynolds stress estimates derived from standard and fast-ping ADCPs. J. Atmos. Oceanic Technol., 23, 854-861, doi:10.1175/ JTECH1885.1.

Osborn, T. R., 1974: Vertical profiling of velocity microstructure. J. Phys. Oceanogr., 4, 109-115, doi:10.1175/ 1520-0485(1974)004<0109:VPOVM>2.0.CO;2.

- 1980: Estimates of local rate of vertical diffusion from dissipation measurements. J. Phys. Oceanogr., 10, 83-89, doi:10.1175/ 1520-0485(1980)010<0083:EOTLRO > 2.0.CO;2.

Price, J. F., R. A. Weller, and R. Pinkel, 1986: Diurnal cycling: Observations and models of the upper ocean response to diurnal heating, cooling, and wind mixing. J. Geophys. Res., 91, 84118427, doi:10.1029/JC091iC07p08411.

Rippeth, T. P., J. H. Simpson, E. Williams, and M. E. Inall, 2003: Measurement of the rates of production and dissipation of turbulent kinetic energy in an energetic tidal flow: Red Wharf Bay revisited. J. Phys. Oceanogr., 33, 1889-1901, doi:10.1175/ 1520-0485(2003)033<1889:MOTROP>2.0.CO;2. 
Sakamoto, K., and K. Akitomo, 2008: The tidally induced bottom boundary layer in a rotating frame: Similarity of turbulence. J. Fluid Mech., 615, 1-25, doi:10.1017/S0022112008003340.

Soulsby, R. L., 1990: Tidal-current boundary layers. Ocean Engineering Science, Parts $A$ and B, B. L. Mehaute and D. M. Hanes, Eds., The Sea-Ideas and Observations on Progress in the Study of the Seas, Vol. 9, John Wiley and Sons, 523-566.

Stacey, M. T., S. G. Monismith, and J. R. Burau, 1999: Measurements of Reynolds stress profiles in unstratified tidal flow. J. Geophys. Res., 104, $10993-10$ 949, doi:10.1029/ 1998JC900095.

Thorpe, S. A., 2007: An Introduction to Ocean Turbulence. Cambridge University Press, 240 pp.
Yoshikawa, Y., T. Matsuno, K. Marubayashi, and K. Fukudome, 2007: A surface velocity spiral observed with ADCP and HF radar in the Tsushima Strait. J. Geophys. Res., 112, C06022, doi:10.1029/2006JG000294.

— T. Endoh, T. Matsuno, T. Wagawa, E. Tsutsumi, H. Yoshimura, and Y. Morii, 2010: The turbulent bottom Ekman boundary layer measured over a continental shelf. Geophys. Res. Lett., 37, L15605, doi:10.1029/2010GL044156.

T. Matsuno, T. Wagawa, T. Hasegawa, K. Nishiuchi, K. Okamura, H. Yoshimura, and Y. Morii, 2012: Tidal and low-frequency currents along the CK line (31 deg $45 \mathrm{~min} \mathrm{~N}$ ) over the East China Sea shelf. Cont. Shelf Res., 50-51, 41-53, doi:10.1016/j.csr.2012.10.007. 